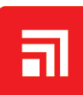 CHITKARA \\ Journal of Nuclear Physics, Material Sciences, Radiation and Applications \\ Journal homepage: https://jnp.chitkara.edu.in/
}

\section{Impact of Nuclear Deformation on Neutron Dripline Prediction: A Study of Mg Isotopes}

\author{
Pankaj Kumar* iD, Virender Thakur iD and Shashi K. Dhiman iD \\ Himachal Pradesh University, Shimla, Himachal Pradesh-171005, India \\ "pankajdhiman659@gmail.com (Corresponding Author)
}

\section{ARTICLE INFORMATION}

Received: February 5, 2021

Accepted: April 15, 2021

Published Online: August 31, 2021

\section{Keywords:}

Relativistic Hartree-Bogoliubov, Quadrupole

deformation, Shape coexistence, Dripline nuclei

DOI: $10.15415 /$ jnp.2021.91004

\section{ABSTRACT}

We have employed the relativistic Hartree-Bogoliubov (RHB) model with density-dependent mesonexchange interaction and separable pairing to investigate neutron dripline mechanisms for heavy $\mathrm{Mg}$ isotopes. In the present study, ${ }^{40} \mathrm{Mg}$ is predicted to be dripline nuclei. The calculations are carried out by taking axial deformation into account. An investigation of shape transition is also done for even-even ${ }^{32-42} \mathrm{Mg}$ isotopes. Our prediction for neutron dripline for ${ }^{40} \mathrm{Mg}$ is consistent with some recent studies.

\section{Introduction}

The study of nuclei far from $\beta$-stability line is a devoted part of modern nuclear-structure studies. The limit of nuclear existence is reached while going away from the stability line. Nuclear drip lines form the edges of the nuclear chart and are defined as limits beyond which a single nucleon become unbound in the nuclear ground-state. The advancement in Radioactive Ion Beam (RIB) facilities and sensitive detection technologies have made it accessible to reach neutron drip lines experimentally. Although the construction of RIBs brings the goal closer, the nuclear drip lines have not been fully mapped experimentally. Moreover, the theoretical location of the nuclear drip line is uncertain $[1,2]$. According to a recent study done by Tsunoda et al. [3], the mechanism responsible for the drip line is related to deformation. However, the neutrons can't be added continuously as the atomic nucleus is characterized by a specific number of protons and neutrons and is bounded by drip lines [4]. Earlier, it was assumed that the strong nuclear force for pn symmetry governed the neutron drip line. But protons and neutrons jump from shell to shell, as they do not stay in a single shell, and move collectively by forming different configurations. These configurations produce deformed shapes. Motivating from this idea, we have done a drip line study of $\mathrm{Mg}$ isotopes using relativistic HartreeBogoliubov (RHB) model with density-dependent meson exchange (DD-ME2) interaction. Our results for groundstate properties and drip line prediction are consistent with recent theoretical studies. For $\mathrm{Mg}$ isotopes, different predictions for neutron drip lines have been proposed. According to the study done in Refs. [5, 6], the heaviest magnesium isotope observed is ${ }^{40} \mathrm{Mg}$ while, the dripline is predicted at ${ }^{42} \mathrm{Mg}$ in some recent studies [3, 7].

Nuclear density functional theories have been using to understand nuclear many-body dynamics for an appreciable description of nuclei near the drip lines [8-11]. It is also found to provide appreciable results for the shape of the nuclei. For the calculations of the shape evolution and ground-state properties, we have used the DIRHBZ numerical code developed by Niksic and others [12]. The present calculations infer the neutron drip line for ${ }^{40} \mathrm{Mg}$.

\section{Theoretical Framework}

Self-consistent mean-field (SCMF) models provide a very successful tool to study nuclear ground-state and excited-state properties from the valley of $\beta$-stability to the nuclear drip-lines throughout the Segre chart (nuclear chart). These models are based on the nuclear energy density functionals (EDF) in which the nucleons are treated as independent particles moving inside the nucleus under the influence of potentials that are derived from such functionals [13]. 


\subsection{Meson Exchange Models}

The detailed description of density dependent meson exchange (DD-ME2) interaction can be found in Ref. [14]. Here, we provide a brief outline of DD-ME2 interaction. The total Lagrangian density of mesons exchange model can be written as:

$$
\begin{aligned}
\mathcal{L} & =\sum_{i} \bar{\psi}_{i}\left(i \gamma_{\mu} \partial^{\mu}-m\right) \psi_{i}+\frac{1}{2} \partial_{\mu} \sigma \partial^{\mu} \sigma-\frac{1}{2} m_{\sigma}^{2} \sigma^{2}-\frac{1}{2} \Omega_{\mu \nu} \Omega^{\mu \nu} \\
& +\frac{1}{2} m_{\omega}^{2} \omega_{\mu} \omega^{\mu}-\frac{1}{4} \overrightarrow{\mathrm{R}}_{\mu \nu} \overrightarrow{\mathrm{R}}^{\mu \nu}+\frac{1}{2} m_{\rho}^{2} \vec{\rho}_{\mu} \cdot \vec{\rho}^{\mu}-\frac{1}{4} \mathrm{~F}_{\mu \nu} F^{\mu \nu} \\
& -g \sigma \bar{\psi} \psi \sigma-g_{\omega} \bar{\psi} \gamma^{\mu} \psi \omega_{\mu}-g_{\rho} \bar{\psi} \vec{\tau} \gamma^{\mu} \psi \cdot \vec{\rho}_{\mu}-e \bar{\psi} \gamma^{\mu} \psi A_{\mu},
\end{aligned}
$$

where $\mathrm{m}$ denotes the bare mass of nucleon and $\psi$ denotes the Dirac spinors. $m_{\sigma}, m_{\omega}, m_{\rho}$ are masses of $\sigma, \omega, \rho$ mesons with corresponding coupling constants $g_{\sigma}, g_{\omega^{\prime}} g_{\rho}$ for the mesons to the nucleons, respectively. $\Omega_{\mu \nu}, \vec{R}_{\mu \nu}, F_{\mu \nu}$ are field tensor of the vector fields $\omega, \rho$, and the photon. Arrows denote isovectors and boldface symbols are used for vectors in ordinary space.

The functionals are described by density-dependent coupling constants $g_{i}(\rho)$ (for $i=\sigma, \omega, \rho, \delta$ ). The coupling of $\sigma$ field and $\omega$ field to the nucleon field is given by [15-17]

$$
\mathrm{g}_{\mathrm{i}}(\rho)=\mathrm{g}_{\mathrm{i}}\left(\rho_{\text {sat }}\right) \mathrm{f}_{\mathrm{i}}(\mathrm{x}) \quad \text { for } \mathrm{i}=\sigma, \omega
$$

with

$$
f_{i}(x)=a_{i} \frac{1+b_{i}\left(x+d_{i}\right)^{2}}{1+c_{i}\left(x+d_{i}\right)^{2}}
$$

which is a function of $x=\rho / \rho_{\text {sat }}$. Here, $\rho_{\text {sat }}$ is the baryon density at saturation in symmetric nuclear matter.

For density dependence of $\rho$-meson coupling, DiracBrueckner suggested the functional form [18], given by

$$
g_{\rho}(\rho)=g_{\rho}\left(\rho_{s a t}\right) e^{-a_{\rho}(x-1)}
$$

The isovector channel is parameterized by $g_{\rho}(\rho)$ and $a_{\rho}$.

\subsection{Relativistic Hartree-Bogoliubov Approximation with a Separable Pairing Interaction}

The inclusion of pairing correlations are important for a quantitative description of open shell nuclei $[19,20]$. The RHB model gives a unified description of particle-hole (ph) and particle-particle (pp) correlations on a mean-field level by using the average self-consistent mean-field potential that encloses the long-range ph correlations and a pairing field potential which sums up the pp correlations. The density matrix in the presence of pairing can be generalized to two densities, the normal density $\hat{\rho}$, and pairing tensor $\hat{k}$. The relativistic Hartree-Bogoliubov energy density functional can be written as:

$$
E_{R H B}[\hat{\rho}, \hat{k}]=E_{R M F}[\hat{\rho}]+E_{\text {pair }}[\hat{k}],
$$

where $E_{R M F}[\hat{\rho}]$ is the nuclear energy density functional and is given by

$$
E_{R M F}\left[\psi, \bar{\psi}, \sigma, \omega^{\mu}, \vec{\rho}^{\mu}, A^{\mu}\right]=\int d^{3} r \mathcal{H}(r)
$$

The pairing part of RHB functional is given by

$$
E_{\text {pair }}[\hat{k}]=\frac{1}{4} \sum_{n_{1} n_{1} n_{2} n_{2}^{\prime}} k_{n_{1} n_{1}^{\prime}}^{*}\left\langle n_{1} n_{1}^{\prime}\left|V^{P P}\right| n_{2} n_{2}^{\prime}\right\rangle k_{n_{2} n_{2}^{\prime}},
$$

where $\left\langle n_{1} n_{1}^{\prime}\left|V^{P P}\right| n_{2} n_{2}^{\prime}\right\rangle$ are the matrix elements of the twobody pairing interaction, and indices $n_{1}, n_{1}^{\prime}, n_{2}$ and $n_{2}^{\prime}$ denote quantum numbers that specify the Dirac indices of the spinor.

The pairing force is separable in momentum space and in r-space has the form of

$$
V^{P P}\left(r_{1}, r_{2}, r_{1}^{\prime}, r_{2}^{\prime}\right)=-G \delta\left(R-R^{\prime}\right) P(r) P\left(r^{\prime}\right),
$$

where $R=\frac{1}{\sqrt{2}}\left(r_{1}+r_{2}\right)$ and $r=\frac{1}{\sqrt{2}}\left(r_{1}-r_{2}\right)$ represent the center of mass and the relative coordinates, respectively and the form factor $P(r)$ is of Gaussian form written as,

$$
P(r)=\frac{1}{\left(4 \pi a^{2}\right)^{3 / 2}} e^{-r^{2} / 2 a^{2}} .
$$

The two parameters ' $G$ ' and 'a' have been adjusted to reproduce the density dependence of gap at the Fermi surface. In the present work, the values of these parameters are taken as $\mathrm{G}=728 \mathrm{MeV} \mathrm{fm}{ }^{3}$ and $\mathrm{a}=0.644 \mathrm{fm}$ for protons and neutrons. They are calculated with the DIS Gogny force in Refs. [19, 21-23]. The pairing force has a finite range and also it conserves translational invariance due to the presence of the factor $\delta(R-R)$. Finally, the pairing energy in the nuclear ground-state is given by

$$
E_{\text {pair }}=-G \sum_{N} P_{N}^{*} P_{N}
$$

\section{Results and Discussion}

Shape is among the most fundamental properties of nuclei and is reflected by the value of the quadrupole deformation parameter. In the axially symmetric case, the deformed 
nuclei can be classified depending upon the value of quadrupole deformation parameter $\left(\beta_{2}\right)$. A positive value of $\beta_{2}$ corresponds to the prolate shape and the negative value of $\beta_{2}$ reflects oblate and $\beta_{2}=0$ corresponds to spherical shape. Fig. 1 presents the potential energy curves (PECs) for ${ }^{32-42} \mathrm{Mg}$ isotopes calculated by constraining the axial deformation parameter. The nuclei ${ }^{32} \mathrm{Mg}$ have a spherical shape which is due to the presence of $N=20$ shell-closure. It can be observed from the figure that almost all neutronrich $\mathrm{Mg}$ isotopes have a prolate shape. The deformation is observed to increase as one move towards heavier isotopes and become saturate and then decreases for ${ }^{42} \mathrm{Mg}$.

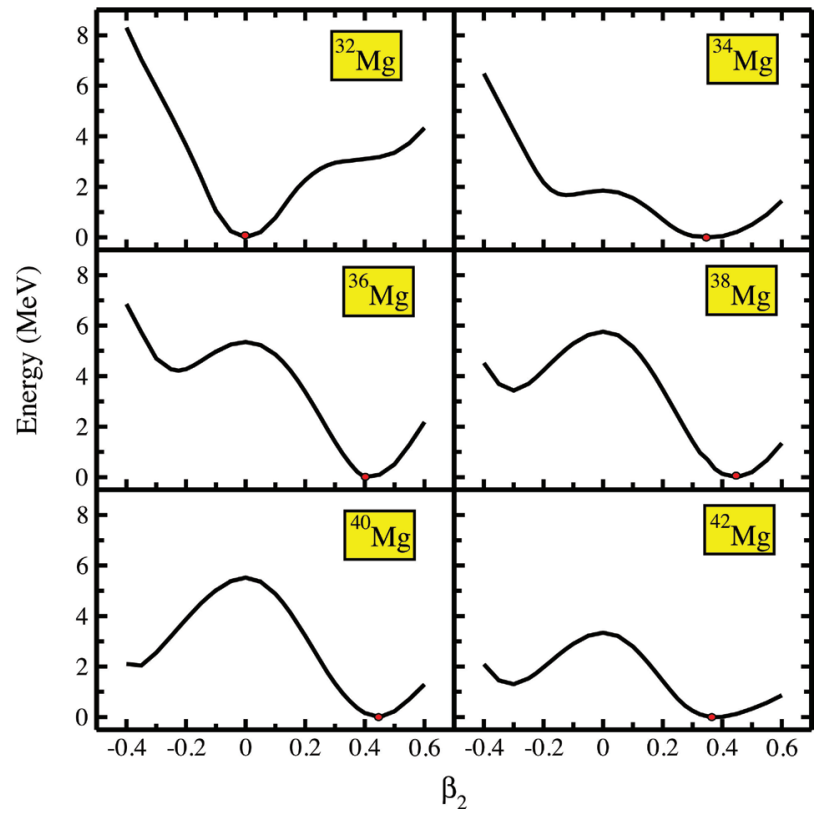

Figure 1: The potential energy curves (PEC), as a function of $\beta_{2}$, for ${ }^{32}{ }^{42} \mathrm{Mg}$ isotopes. The energies are normalized with respect to the binding energy of the global minima.

In Fig. 2, we plot the binding energy per nucleon E/A, the neutron chemical potential $\lambda_{n}$, the ground-state quadrupole deformation parameter $\left(\beta_{2}\right)$, and two-neutron separation energies $S_{2 \mathrm{n}}$ for neutron-rich $\mathrm{Mg}$ isotopes as function of the mass number A. In the top panel of Fig. 2, we observed a decrease in the $\mathrm{E} / \mathrm{A}$ value indicating the loosely bound behavior of heavier $\mathrm{Mg}$ isotopes. In panel (b) of Fig. 2, the neutron chemical potential $\lambda_{n}$ is plotted as a function of $A$. The systematics of $\lambda_{n}$ can be related to the large deformation. The negative value of chemical potential assures that an even-N system is bound. The positive value of $\lambda_{n}$ for ${ }^{42} \mathrm{Mg}$ indicates an unbound system. The ground-state quadrupole deformation parameter, obtained from PECs, for ${ }^{32-42} \mathrm{Mg}$ can be depicted in panel (c) of Fig. 2. Moving away from $N=20$ shell closure, all the neutron-rich $\mathrm{Mg}$ isotopes have a prolate shape. The primary factor that determines the stability of the particle and the nucleoid drip line is two-neutron separation energy $S_{2 n}$ [4]. It is defined as the amount of energy needed to remove two neutrons. The stability and instability of the nucleus to nucleon emission can be related to the positive value of $S_{2 n}$ and negative value of $S_{2 n}$ respectively. The drip line is reached when $S_{2 n} \approx 0$. In the bottom panel of Fig. 2, we compared the results of two-neutron separation energies $S_{2 n}$, calculated with difference in binding energies $\left(S_{2 n}(Z, N)\right.$ $\left.=E_{b}(Z, N)-E_{b}(Z, N-2)\right)$ and with the related quantity $\left(S_{2 n}=-2 \lambda_{n}\right)$ [4]. A negative value of $S_{2 n}$ for ${ }^{42} \mathrm{Mg}$ shows it to be an unbound nucleus.

The variation of nuclear shape and ground-state energy are presented, as a function of neutron number $(\mathrm{N})$, in Fig. 3. The nucleus ${ }^{32} \mathrm{Mg}$ has a spherical shape and have lower binding energy. The evolution of ellipsoidal (prolate in the present case) deformation is observed with the addition of the number of neutrons and an increase in binding energy is seen from ${ }^{32} \mathrm{Mg}$ to ${ }^{40} \mathrm{Mg}$. With the increase in neutron number, the superposition of single-particle states of $p f$ shell leads to enhance the deformation, giving the nucleus additional binding energy. At $N=28$, this additional binding energy saturates and decreases after adding a pair of neutron to the nucleus. Thus, after this point, the deformation gets weaker by the addition of two neutrons and they drip off the nuclei.

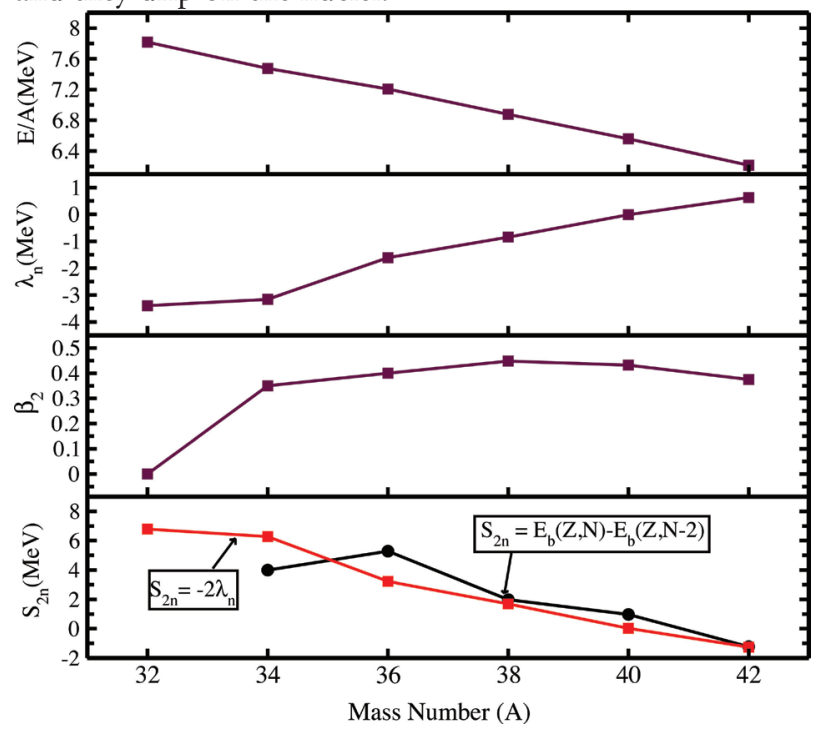

Figure 2: Binding energies per nucleon $\mathrm{E} / \mathrm{A}$, neutron chemical potentials $\lambda_{\mathrm{n}}$, quadrupole deformation parameter $\left(\beta_{2}\right)$, and twoneutron separation energies $S_{2 n}$ for neutron-rich $\mathrm{Mg}$ isotopes plotted as function of the mass number A.

\section{Conclusion}

In this paper, we have performed axially constrained selfconsistent calculations using relativistic Hartree-Bogoliubov (RHB) model with density-dependent meson-exchange 
interaction (DD-ME2) and with separable pairing. We have studied the shape evolution of some neutron-rich $\mathrm{Mg}$ isotopes and calculated their ground-state properties. The negative value of two-neutron separation energies for ${ }^{42} \mathrm{Mg}$ correspond to an unbound system. The heaviest isotope that is bound against two neutron decay, based on positive $S_{2 n}$ is ${ }^{40} \mathrm{Mg}$. Our calculations predict ${ }^{40} \mathrm{Mg}$ to be a drip line nucleus, which is consistent with recent theoretical predictions. The influence of deformation for the prediction of neutron drip line for $\mathrm{Mg}$ isotopes is also studied.

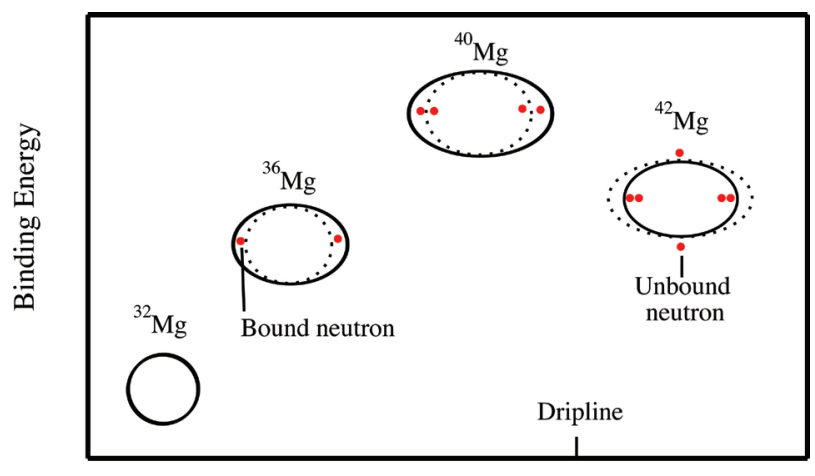

$\mathrm{N}$

Figure 3: Variation of nuclear shape and ground-state energy, as a function of neutron number $(\mathrm{N})$.

\section{Acknowledgements}

One of the authors, Mr. Pankaj Kumar, acknowledges the financial assistance Senior Research Fellowship provided by Council of Scientific and Industrial Research (CSIR), New Delhi vide reference no. 09/237(0165)/2018-EMR-I.

\section{References}

[1] A. V. Afanasjev, S. E. Agbemava, D. Ray and P. Ring, Phys. Rev. C 91, 014324 (1992). https://doi.org/10.1103/PhysRevC.91.014324

[2] L. Neufcourtet al., Phys. Rev. Lett. 122, 062502 (2019). https://doi.org/10.1103/PhysRevLett.122.062502

[3] N. Tsunoda et al., Nature 587, 66 (2020). https://doi.org/10.1038/s41586-020-2848-x

[4] J. Erler et al., Nature 486, 509 (2012). https://doi.org/10.1038/nature11188

[5] T. Baumann, et al., Nature 449, 1022 (2007). https://doi.org/10.1038/nature06213

[6] H.L. Crawford et al., Phys. Rev. Lett 122, 052501 (2014). https://doi.org/10.1103/PhysRevLett.122.052501
[7] Q. Chai, J. Pei, N. Fei and D. Guan, Phys. Rev. C 102, 014312 (2020).

https://link.aps.org/doi/10.1103/PhysRevC.102.014312

[8] V. Thakur and S. K. Dhiman, Nucl. Phys. A 992, 121623 (2019).

https://doi.Org/10.1016/j.nuclphysa.2019.121623

[9] V. Thakur Nuet et al., Nucl. Phys. A 1002, 121981 (2020).

https://doi.Org/10.1016/j.nuclphysa.2020.121981

[10] P. Kumar and S. K. Dhiman, Nucl. Phys. A 1001, 121935 (2020).

https://doi.Org/10.1016/j.nuclphysa.2020.121935

[11] P. Kumar et al., Eur. Phys. J. A 57, 36 (2021). https://doi.org/10.1140/epja/s10050-021-00346-6

[12] T. Niksic, N. Paar, D. Vretenar and P. Ring, Computer Physics Communications 185, 1808 (2014). https://doi.Org/10.1016/j.cpc.2014.02.027

[13] M. Bender, P.-H. Heenen and P.-G. Reinhard, Rev. Mod. Phys. 75, 121 (2003).

https://doi.org/10.1103/RevModPhys.75.121

[14] G. Lalazissis, T. Niksic, D. Vretenar and P. Ring, Phys. Rev. C 71, 024312 (2005). https://doi.org/10.1103/PhysRevC.71.024312

[15] S. Typel and H. H. Wolter, Nucl. Phys. A 656, 331 (1999). https://doi.org/10.1016/S0375-9474(99)00310-3

[16] F. Hofmann, C. Keil and H. Lenske, Phys. Rev. C 64, 034314 (2001). https://doi.org/10.1103/PhysRevC.64.034314

[17] T. Niksic, D. Vretenar, P. Finelli and P. Ring, Phys. Rev. C 66, 024306 (2002). https://doi.org/10.1103/PhysRevC.66.024306

[18] F. De Jong and H. Lenske, Phys. Rev. C 57, 3099 (1998). https://doi.org/10.1103/PhysRevC.57.3099

[19] Y. Tian, Z.-Y. Ma and P. Ring, Phys. Lett. B 676, 44 (2009). https://doi.org/10.1016/j.physletb.2009.04.067

[20] T. Niksic, P. Ring, D. Vretenar, Y. Tian and Z.-Y. Ma, Phys. Rev. C 81, 054318 (2010). https://doi.org/10.1103/PhysRevC.81.054318

[21] Y. Tian, Z.-Y. Ma and P. Ring, Phys. Rev. C 79, 064301 (2009). https://doi.org/10.1103/PhysRevC.79.064301 


\section{药 \\ CHITKARA}

Journal of Nuclear Physics, Material Sciences, Radiation and Applications

Chitkara University, Saraswati Kendra, SCO 160-161, Sector 9-C, Chandigarh, 160009, India

Volume 9, Issue 1

August 2021

ISSN 2321-8649

Copyright: [C 2021 Pankaj Kumar, Virender Thakur and Shashi K. Dhiman] This is an Open Access article published in Journal of Nuclear Physics, Material Sciences, Radiation and Applications (J. Nucl. Phy. Mat. Sci. Rad. A.) by Chitkara University Publications. It is published with a Creative Commons Attribution- CC-BY 4.0 International License. This license permits unrestricted use, distribution, and reproduction in any medium, provided the original author and source are credited. 